\title{
Biosynthesis of silver nanoparticles using lemon leaves extract and its application for antimicrobial finish on fabric
}

\author{
Padma S. Vankar • Dhara Shukla
}

Received: 24 November $2011 /$ Accepted: 16 December 2011/Published online: 28 December 2011

(C) The Author(s) 2011. This article is published with open access at Springerlink.com

\begin{abstract}
Preparation of silver nanoparticles have been carried out using aqueous extract of lemon leaves (Citrus limon) which acts as reducing agent and encapsulating cage for the silver nanoparticles. These silver nanoparticles have been used for durable textile finish on cotton and silk fabrics. Remarkable antifungal activity has been observed in the treated fabrics. The antimicrobial activity of silver nanoparticles derived from lemon leaves showed enhancement in activity due to synergistic effect of silver and essential oil components of lemon leaves. The present investigation shows the extracellular synthesis of highly stable silver nanoparticles by biotransformation using the extract of lemon leaves by controlled reduction of the $\mathrm{Ag}^{+}$ion to $\mathrm{Ag}^{0}$. Further the silver nanoparticles were used for antifungal treatment of fabrics which was tested by antifungal activity assessment of textile material by Agar diffusion method against Fusarium oxysporum and Alternaria brassicicola. Formation of the metallic nanoparticles was established by FT-IR, UV-Visible spectroscopy, transmission electron microscopy, scanning electron microscopy, atomic force microscopy.
\end{abstract}

Keywords Silver nanoparticles - Citrus limon leaves · Antifungal activity $\cdot$ Cotton $\cdot$ Silk

\section{Introduction}

Nanoparticles using plant extracts have received attention in the recent times as it is a simple and economical method.

P. S. Vankar $(\bowtie) \cdot$ D. Shukla

Facility for Ecological and Analytical Testing, (FEAT), Indian Institute of Technology, 204 'A' Southern Block, Kanpur 208 016, India

e-mail: psv@iitk.ac.in
Jose-Yacaman et al. reported the formation of gold and silver nanoparticles by living plants for the first time (GardeaTorresdey et al. 2002, 2003). Sastry et al. attained the biosynthesis of metal nanoparticles by plant leaf extracts and explored their potential applications (Shankar et al. 2003a, b). They studied bioreduction of chloroaurate ions or silver ions by a broth of geranium leaves as well as Neem leaves (Shankar et al. 2004a). Further, they also explored the mechanism of formation of gold nanotriangles by lemongrass extracts. They found that the nanotriangles seemed to grow by a process involving rapid reduction, assembly and roomtemperature sintering of 'liquid-like' spherical gold nanoparticles (Shankar et al. 2004b). They had also synthesized gold nanotriangles using Tamarind leaf extract and studied their potential application in vapor sensing (Ankamwar et al. 2005). Very recently, Sastry et al. had demonstrated synthesis of gold nanotriangles and silver nanoparticles using Aloe vera plant extract (Chandran et al. 2006).

\section{Experimental}

\section{Materials}

Dark green Citrus limon (Lemon) leaves were collected from IIT, Kanpur and used for generating silver nanoparticles. Two pure fungal strains, viz. Fusarium oxysporum, Alternaria brassicicola, were procured from MTCC section of Indian Institute of Pulse Research, Kanpur, India.

\section{Media and chemicals}

Readymade potato dextrose agar (PDA) of Himedia make was used to maintain as well as to propagate the fungal culture. Peptone and Dextrose were used to make medium 
for observing fungal growth in broth. Silver nitrate $\left(\mathrm{AgNO}_{3}\right)$ salt was purchased from Spectrochem, Kanpur. Methanol and other chemicals were of Rankem (Ranbaxy) make.

\section{Preparation of bioextract}

Twenty grams fresh leaves of lemon were washed with tap water and then washed with distilled water, air dried and then they were finely cut and soaked in $100 \mathrm{ml}$ boiling distilled water for 5-10 min and filtered through Whatman filter paper no. 42. This extract was used for generating silver nanoparticles. This bioextract is always used fresh.

Preparation of silver nanoparticles (SNP) using bioextract

Five milliliters of leaves extract was added into $45 \mathrm{ml}$ $0.002 \mathrm{M} \mathrm{AgNO}_{3}$ solution in $100 \mathrm{ml}$ conical flasks at room temperature in dark. After $1 \mathrm{~h}$, formation of silver particles started to appear in the flask.

UV-Visible spectral analysis

The bioreduction of $\mathrm{Ag}^{+}$in aqueous solution was monitored by periodic sampling of aliquots $(0.2 \mathrm{ml})$ of the suspension, then diluting the samples with $2 \mathrm{ml}$ of deionized water and subsequently measuring UV-Visible spectra of the resulting diluents. UV-Visible spectroscopy analyses of silver nanoparticles produced were carried out as a function of time needed for bioreduction at room temperature on Thermo He $\lambda$ ios $\alpha$ model spectrophotometer at a resolution of $1 \mathrm{~nm}$.

Fourier Transform-infrared spectral analysis

The residual solution containing the nanoparticles was centrifuged at 4,800 rpm for $10 \mathrm{~min}$ and the resulting suspension was redispersed in $20 \mathrm{ml}$ sterile distilled water. The centrifuging and redispersing process was repeated thrice. Thereafter, the purified suspension was completely dried at $60^{\circ} \mathrm{C}$. Finally, the dried nanoparticles were analyzed by Vertex 70 model of Bruker for FTIR.

TEM and AFM observation of silver nanoparticles

The centrifuged and redispersed suspension was sampled for TEM analysis. TEM samples of the aqueous suspension of silver nanoparticles were prepared by placing a drop of the suspension on carbon-coated copper grids and by allowing water to evaporate. TEM data were collected on FEI TECNAI 02 Machine having software TECNAI $\mathrm{G}^{2}$. AFM data were collected on Molecular Imaging Agilent
Machine and pictures were collected on PicoScan software. Cantilevers $\mu$ Masch $(\mathrm{Cu}-\mathrm{Au})$ with Tip curvature less than $10 \mathrm{~nm}$ were used in Molecular Imaging probe.

Premier ColorScan machine was used for shade change and Lab values in SNP dyed cotton and silk. SEM micrographs were taken on FEI Quanta 200.

Plating potato dextrose Agar

PDA was accurately weighed and dissolved in distilled water, then kept in conical flask and plugged with cotton before keeping it for sterilization. After sterilization of about $20 \mathrm{ml}$ Agar was poured in each sterilized petri plates then these plates were allowed to cool so that agar gets solidified and then inoculation was done.

Dyeing of cotton and silk by silver nanoparticles

Pre-washed cotton and silk fabrics dyed with lemon leaf extract were used as control fabric whereas silver nanoparticles-treated cotton and silk pieces were used as sample fabrics to assess durable textile finishing by subsequent washing method and further for antifungal activity. The control samples were prepared by dipping fabrics in $20 \%$ aqueous extract of lemon leaves at $65-70^{\circ} \mathrm{C}$ for $2 \mathrm{~h}$ keeping material to liquor ratio 1:25. Then it was dried in shade without squeezing. Similarly pre-washed cotton and silk were dipped in silver nanosolution generated by lemon leaves, for $4 \mathrm{~h}$ and then taken out and dried in shade. The cotton-treated fabric was grayish brown and silk-treated fabric was greenish brown in color.

\section{Durable textile finish test}

The pieces of cotton and silk dyed/coated with silver nanoparticles having dimensions of $3 \times 2.5 \mathrm{~cm}$ (length $\times$ width) were used for wash sustainability to assess the results of durable textile finish. Five subsequent washings were carried out. Washings were carried out by thorough wetting of treated fabrics in distilled water where samples were left for $4 \mathrm{~h}$ at room temperature. After drying, changes in sample color and bleeding to white fabric were determined. Theses samples were further used for estimation of antifungal activity.

Antifungal activity assessment of textile material

\section{Parallel streak method}

In this method as well, control and sample fabric pieces were placed with intimate contact of the media, i.e., PDA which had been previously streaked with an inoculums $(0.05 \mathrm{ml})$ of test organism. After $18-24 \mathrm{~h}$, a streak of uninterrupted or 
low-colony area was counted along the side of fabric indicating antifungal effectiveness of the fabric.

\section{Result and discussion}

Biosynthesis of silver nanoparticles by lemon leaves extract

It is well known that silver nanoparticles exhibit yellowishbrown color in aqueous solution due to excitation of surface plasmon vibrations in silver nanoparticles (Shankar et al. 2004a, b; Ankamwar et al. 2005; Chandran et al. 2006). Reduction of the silver ion to silver nanoparticles during exposure to the plant leaf extracts was followed by color change and as well as by UV-Vis spectroscopy. It is generally recognized that $\mathrm{UV}-\mathrm{V}$ isible spectroscopy could be used to examine size- and shape-controlled nanoparticles in aqueous suspensions. UV-Visible spectra that were recorded at different intervals for monitoring the reaction, the appearance of a surface plasmon resonance (SPR) band increased in intensity with time. It also reveals the production of silver nanoparticles within $1 \mathrm{~h}$. Figure 1a shows the UV-Visible absorption spectra recorded from the silver nanoparticles solution after $2.5 \mathrm{~h}$ of reaction (curve A) and the lemon leaves extract (curve B). Gold nanoparticles from Mirabilis flowers were analyzed similarly by Vankar and Bajpai 2010.

FT-IR absorption spectra of the dried biomass of lemon leaves before and after bioreduction, as shown in Fig. 1b, the information regarding the chemical change of the functional groups involved in bioreduction can be assessed. The band at $1,101 \mathrm{~cm}^{-1}$ which might be contributed by the $-\mathrm{C}-\mathrm{O}$ groups of the polyols such as flavones, terpenoids and polysaccharides in the biomass appeared as a significant peak. FT-IR analysis of the bioextract before and after the addition of silver solution revealed the strong bands at 1,021, 1,443,
1,634 and $3,428 \mathrm{~cm}^{-1}$. The band at $1,021 \mathrm{~cm}^{-1}$ corresponded to $\mathrm{C}-\mathrm{N}$ stretching vibrations of amine. The band at $1,443 \mathrm{~cm}^{-1}$ corresponded to $\mathrm{C}-\mathrm{H}$ and $\mathrm{OH}$ bending and $3,428 \mathrm{~cm}^{-1}$ was attributed to characteristic of $-\mathrm{NH}$ stretching of amide (II) band. The weaker band at $1,634 \mathrm{~cm}^{-1}$ corresponded to amide I, arisen due to carbonyl stretch in proteins.

Scanning electron micrograph (Fig. 2a) of SNP from lemon leaves confirm that they form in large numbers and they are almost uniform in size. Silk and cotton are dyed with this kind of SNPs. This uniformity of size and shape considerably enhance wash fastness of textile and consequently to anti fungal activity of dyed cotton and silk.

The particle size in this TEM image (Fig. 2b) has been found to be in the range between 8 and $15 \mathrm{~nm}$. Specific sizes ascertained were $8.27,13.79$ and $14.48 \mathrm{~nm}$. The particle size from the TEM image (Fig. 2b) was found to be in the range between 15 and $30 \mathrm{~nm}$. Other nanoparticles showed size 19.23, 28.8 and $30 \mathrm{~nm}$ as well.

In Fig. 2c silver nanoparticles have been shown as topographic image. Atomic force microscopy (AFM) showed well-dispersed, heterogeneously-shaped nanoparticles.

\section{Durable textile finish}

Durability and sustainability of the nano finish on cotton and silk has been shown in Tables 1 and 2, respectively, through change in CIEL $a^{*} b^{*}$ values which are internationally accepted values for change in lightness/darkness and color index. The nano-finished fabrics (both cotton and silk) show very small changes in $L$ values even after five washes. The fabric swatches show the same results (Fig. 3a, b). This sustainability of dyed fabric was responsible for antifungal or antimicrobial activity of SNPs dyed cotton and silk as the layer/coating of SNPs on cloth act as shield to restrict fungal growth.

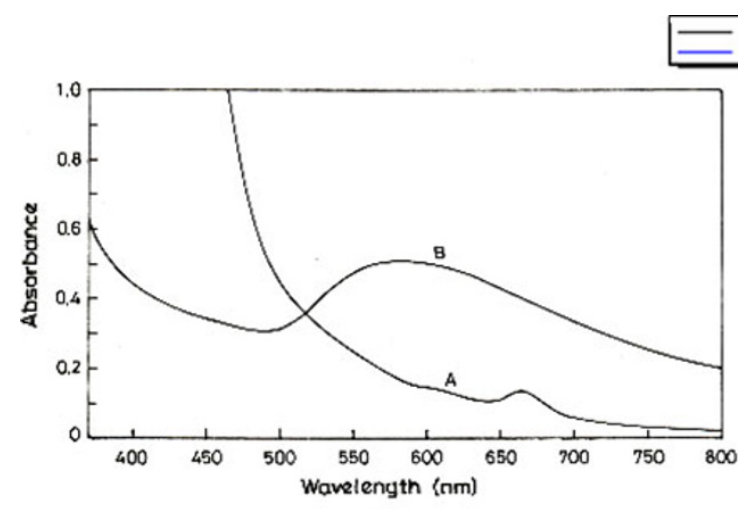

a

Lem on silver $\mathrm{N}$ and

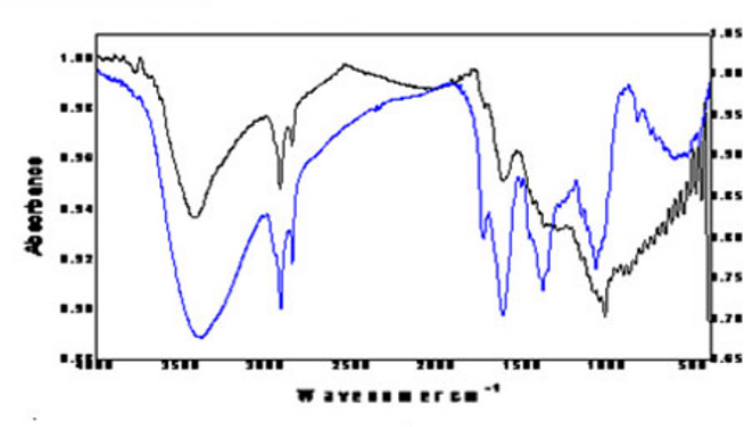

b

Fig. 1 a Visible spectra of silver nanoparticles from lemon leaves extract $(A)$ and lemon leaves extract $(B)$; b FT-IR spectra of silver nanoparticles from lemon leaves extract (Black) and lemon leaves extract (Blue) 


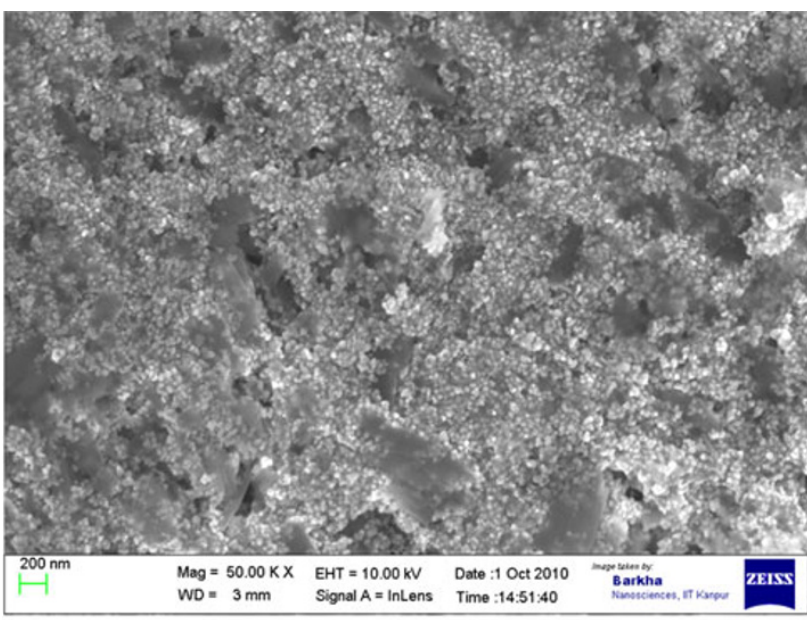

a

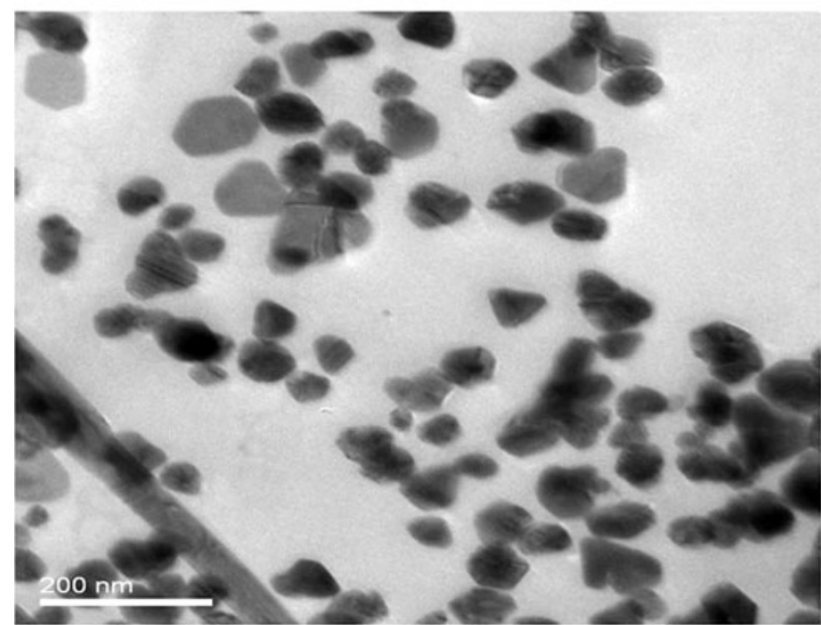

b

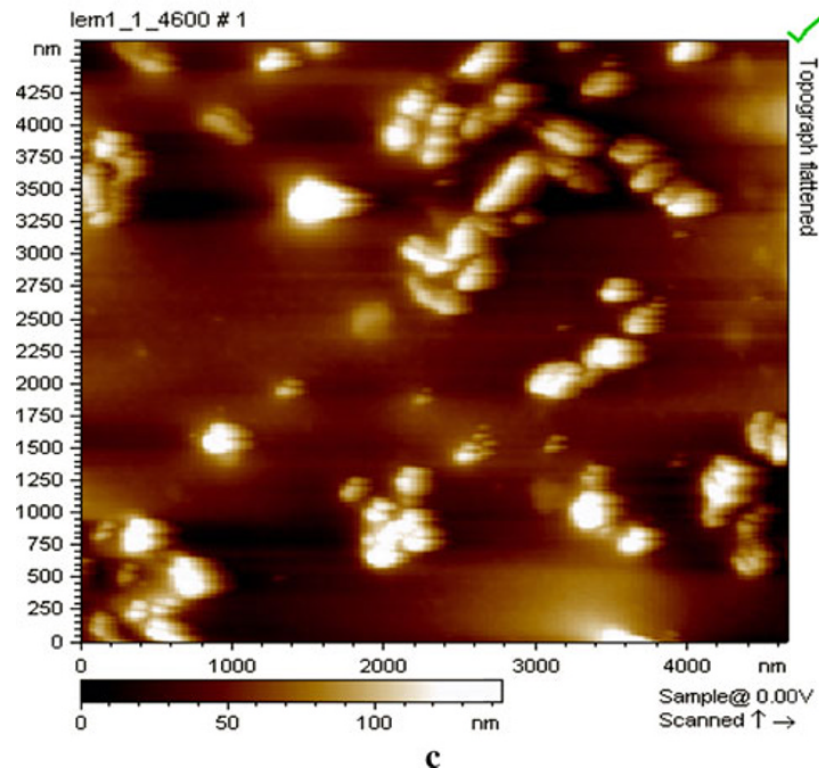

Fig. 2 a SEM, b TEM, c AFM of silver nanoparticles from lemon leaves
Table 1 Wash sustainability of silver nanoparticle-treated cotton

\begin{tabular}{llllllr}
\hline & $L$ & $a^{*}$ & $b^{*}$ & $C$ & $H$ & \multicolumn{1}{l}{$\delta E$} \\
\hline Unwashed & 79.181 & 2.90 & 4.94 & 5.73 & 59.57 & 7.74 \\
Wash I & 79.207 & 2.54 & 4.96 & 5.58 & 62.87 & 7.46 \\
Wash III & 79.379 & 4.88 & 6.19 & 7.88 & 51.68 & 8.64 \\
Wash V & 79.222 & 6.86 & 5.91 & 9.05 & 40.70 & 10.55 \\
\hline
\end{tabular}

Table 2 Wash sustainability of silver nanoparticle-treated silk

\begin{tabular}{llllllr}
\hline & $L$ & $a^{*}$ & $b^{*}$ & $C$ & $H$ & \multicolumn{1}{l}{$\delta E$} \\
\hline Unwashed & 72.817 & 5.18 & 15.54 & 16.38 & 71.53 & 9.16 \\
Wash I & 72.896 & 4.45 & 15.65 & 16.27 & 74.09 & 8.58 \\
Wash III & 72.426 & 6.615 & 14.41 & 15.88 & 65.36 & 10.05 \\
Wash V & 73.044 & 5.28 & 16.21 & 17.05 & 71.92 & 9.59 \\
\hline
\end{tabular}

Antifungal activity of silver nano-dyed fabric

In this experiment, lemon silver nano-dyed cotton and silk fabric pieces were put on Fusarium and Alternaria culture on opposite side and for this, $20 \mathrm{~h}$ old culture was used and inhibition of colony was checked after every $3 \mathrm{~h}$ and compared with the control plate. Substantial inhibition of both the fungal species was obtained in terms of growth restriction in both the fabrics in as shown in Figs. 4, 5.

\section{Mechanism of action}

The mechanism for the antimicrobial action of silver ions is not properly understood; however, the effect of silver ions on microbe can be observed by the structural and morphological changes. It is suggested that when DNA molecules are in relaxed state the replication of DNA can be effectively conducted. But when the DNA is in condensed form it loses its replication ability hence, when the silver ions penetrate inside the microbial cell the DNA molecule turns into condensed form and loses its replication ability leading to cell death. Also, it has been reported that heavy metals react with proteins by getting attached with the thiol group and the proteins get inactivated (Liau et al. 1997; Feng et al. 2000).

The silver nanoparticles show efficient antimicrobial property compared to other salts due to their extremely large surface area, which provides better contact with microorganisms. Silver is inherently anti-microbial and antibacterial substance. By incorporating nanoscale silver into textiles, the manufacturers can make materials that use a small amount of silver to kill the microbes present on the surface of the clothing material, thus can be treated with silver nanoparticles to help prevent spoilage rising from microbial growth in damp areas. Silver nanoparticles have 
Fig. 3 Durable finish by silver nano on (a) cotton with silver nano after wash I, II, III, IV and $\mathrm{V}$ and (b) silver nano silk after wash I, II, III, IV and V
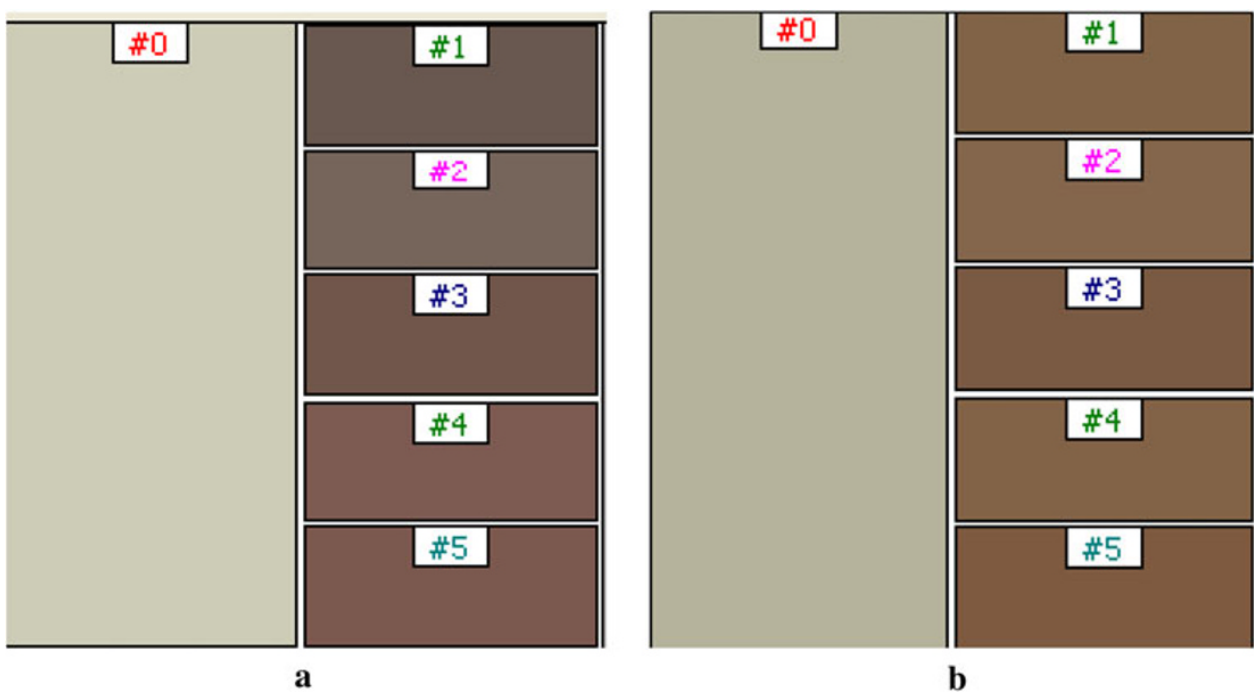

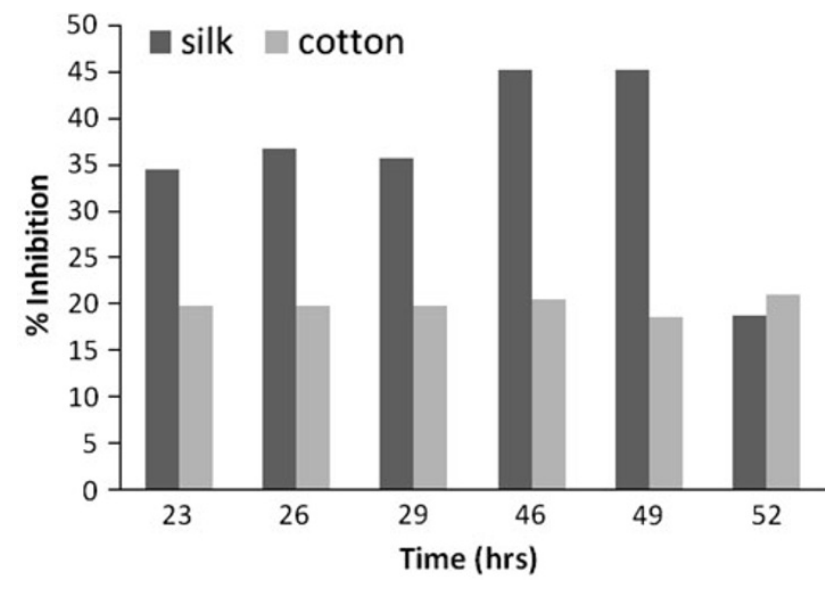

Fig. $4 \%$ Inhibition of Fusarium oxysporum by SNP-dyed fabric

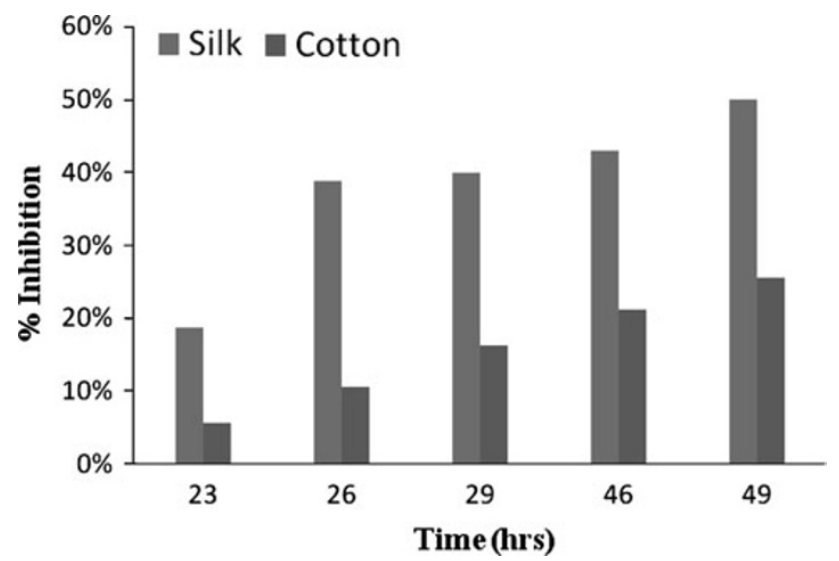

Fig. $5 \%$ Inhibition of Alternaria brassicicola by SNP-dyed fabric

relatively large surface area available, ideally suited for effective control of germs, molds and fungus. Not much is documented about the exact mechanism of antifungal activity of the silver nanoparticles. The antimicrobial activity of silver NP derived from lemon leaves showed enhancement in activity due to synergistic effect of silver and essential oil components of lemon leaves.

\section{Conclusion}

The reduction of silver ions by lemon leaves extract resulted in the formation of stable nanoparticles with multi shaped morphologies resulted in $>100 \mathrm{~nm}$ size range of silver nanoparticles. The rate of reaction for the synthesis of nanoparticles by this method by lemon leaves extract is $2.5 \mathrm{~h}$, which is much faster than the coriander leaf mediated synthesis (12 h) (Narayanan and Sakthivel 2008) and faster than the microbes-mediated synthesis (24-120 h) (Prakash et al. 2010). Silver nanoparticles synthesized by the green chemistry approach reported in this study using lemon leaves extract could have potent applications in biomedical and pharmaceutical applications. Furthermore, it has been demonstrated that use of a natural, renewable and low-cost biological reducing agent, such as lemon leaves can produce metal nanostructures in aqueous solution at ambient temperature, avoiding the presence of hazardous and toxic solvents. The antifungal activity of SNP derived from lemon leaves showed enhancement in activity due to synergistic effect of silver nanoparticles and essential oil components of lemon leaves the effectivity was enhanced as observed from the data.

Acknowledgments One of the authors, Dhara Shukla is thankful to Council of Scientific and Industrial Research, Govt. of India for providing financial support.

Open Access This article is distributed under the terms of the Creative Commons Attribution License which permits any use, distribution and reproduction in any medium, provided the original author(s) and source are credited. 


\section{References}

Ankamwar B, Chaudhary M, Sastry M (2005) Gold nanotriangles biologically synthesized using tamarind leaf extract and potential application in vapor sensing. Synth React Inorg Metal Org Nano Metal Chem 35(1):19-26

Chandran SP, Chaudhary M, Pasricha R, Ahmad A, Sastry M (2006) Synthesis of gold nanotriangles and silver nanoparticles using aloe vera plant extract. Biotechnol Progr 22(2):577-583. doi:10.1021/ bp0501423

Feng QL, Wu J, Chen GQ, Cui FZ, Kim TN, Kim JO (2000) A mechanistic study of the antibacterial effect of silver ions on Escherichia coli and Staphylococcus aureus. J Biomed Mater Res 52(4):662-668. doi:10.1002/1097-4636(20001215)52:4 $<662$ :aid-jbm10>3.0.co;2-3

Gardea-Torresdey JL, Parsons JG, Gomez E, Peralta-Videa J, Troiani HE, Santiago P, Yacaman MJ (2002) Formation and growth of $\mathrm{Au}$ nanoparticles inside live alfalfa plants. Nano Lett 2(4): 397-401

Gardea-Torresdey JL, Gomez E, Peralta-Videa JR, Parsons JG, Troiani H, Jose-Yacaman M (2003) Alfalfa sprouts: a natural source for the synthesis of silver nanoparticles. Langmuir 19(4):1357-1361. doi:10.1021/la020835i

Liau SY, Read DC, Pugh WJ, Furr JR, Russell AD (1997) Interaction of silver nitrate with readily identifiable groups: relationship to the antibacterial action of silver ions. Lett Appl Microbiol 25(4):279-283. doi:10.1046/j.1472-765X.1997.00219.x

Narayanan KB, Sakthivel N (2008) Coriander leaf mediated biosynthesis of gold nanoparticles. Mater Lett 62(30):4588-4590. doi: 10.1016/j.matlet.2008.08.044

Prakash A, Sharma S, Ahmad N, Ghosh A, Sinha P (2010) Bacteria mediated extracellular synthesis of metallic nanoparticles. Int Res J Biotechnol 1(5):71-79

Shankar SS, Ahmad A, Pasricha R, Sastry M (2003a) Bioreduction of chloroaurate ions by geranium leaves and its endophytic fungus yields gold nanoparticles of different shapes. J Mater Chem 13(7): $1822-1826$

Shankar SS, Ahmad A, Sastry M (2003b) Geranium leaf assisted biosynthesis of silver nanoparticles. Biotechnol Progr 19(6):1627-1631. doi:10.1021/bp034070w

Shankar SS, Rai A, Ahmad A, Sastry M (2004a) Rapid synthesis of $\mathrm{Au}, \mathrm{Ag}$, and bimetallic $\mathrm{Au}$ core- $\mathrm{Ag}$ shell nanoparticles using Neem (Azadirachta indica) leaf broth. J Colloid Interf Sci 275(2):496-502. doi:10.1016/j.jcis.2004.03.003

Shankar SS, Rai A, Ankamwar B, Singh A, Ahmad A, Sastry M (2004b) Biological synthesis of triangular gold nanoprisms. Nat Mater 3(7):482-488

Vankar PS, Bajpai D (2010) Preparation of gold nanoparticles from Mirabilis jalapa flowers. Indian J Biochem Biophys 47(3): 157-160 